\title{
Circadian Rhythmicity of Skeletal Muscle Metabolism
}

\author{
An Expert Interview with Patrick Schrauwen
}

Department of Nutrition and Movement Sciences, NUTRIM School of Nutrition and Translational Research in Metabolism Faculty of Health, Medicine and Life Sciences, Maastricht University, Maastricht, the Netherlands

DOI: https://doi.org/10.17925/USE.2019.15.2.57

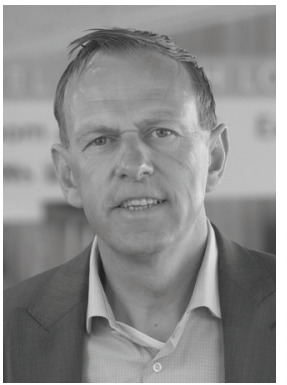

Patrick Schrauwen

Professor Patrick Schrauwen is professor of metabolic aspects of type 2 diabetes mellitus at the NUTRIM school for Nutrition and Translational Research in Metabolism, Maastricht University, the Netherlands. His multidisciplinary team combines cellular and molecular biology techniques and state-of-the-art imaging techniques with human clinical intervention trails. Since 2010, he has been the leader of the division 'Obesity, type 2 diabetes and cardiovascular health' at NUTRIM. In 2016, Prof. Dr Schrauwen was awarded the MINKOWSKI Award of the EASD, the most prestigious European award in diabetes research for researchers $<45$ years old.

\section{Keywords}

Circadian rhythm, insulin sensitivity, skeletal muscle, type 2 diabetes mellitus

Disclosure: Patrick Schrauwen has no conflicts of interest to declare in relation to this article.

Acknowledgment: Medical writing assistance was provided by Dr CG Griffin of Griffin Scientific Ltd on behalf of Touch Medical Media and

was supported by Touch Medical Media.

Review Process: This is an expert interview and as such, has not undergone the journal's standard peer review process

Compliance with Ethics: This article is an expert interview and did not involve any studies with human or animal subjects performed by any of the authors.

Authorship: The named author meets the International Committee of Medical Journal Editors (ICMJE) criteria for authorship of this manuscript, takes responsibility for the integrity of the work as a whole, and has given final approval for the version to be published.

Received: July 16, 2019

Accepted: October 6, 2019

Citation: US Endocrinology. 2019;15(2):57-8

Corresponding Author: Patrick Schrauwen, Department of Nutrition and Movement Sciences,

NUTRIM school for Nutrition and Translational Research in Metabolism, Maastricht University, PO Box 616, Maastricht, The Netherlands. E: p.schrauwen@maastrichtuniversity.nl

Support: No funding was received for the publication of this manuscript.
$\mathrm{T}$ here is increasing evidence to support the association between the circadian clock and the development of metabolic diseases. ${ }^{1}$ Misalignment of circadian rhythm can increase insulin resistance and decrease pancreatic function. ${ }^{2}$ Such disruptions may increase the likelihood of developing type 2 diabetes mellitus (T2DM). ${ }^{1,3}$ The effects of natural rhythm disruptions have been highlighted in increased T2DM risk in people employed in shift work, such as nurses. ${ }^{4}$ Recent research has examined the interplay between circadian rhythm and insulin resistance in skeletal muscle. ${ }^{5}$

\section{Q. What was the rationale for studying the effect of circadian rhythm on skeletal muscle insulin sensitivity?}

We now appreciate the role of diet and exercise in the progression of insulin resistance, but the body's natural rhythms are also a key factor. Skeletal muscle plays a key role in the development of insulin resistance and this study examined the relationship between circadian rhythm and skeletal muscle metabolism. ${ }^{5}$

\section{Q. What are the key findings from these investigations?}

We have learned that mitochondrial function in muscle, which plays a role in insulin resistance development, has a 24-hour rhythm in healthy people. This rhythm may be disrupted in people with prediabetes and with T2DM. Disrupting the normal day-night rhythm — which for example, happens in people who are employed in shift work or an increasingly '24-hour culture'-leads to muscle insulin resistance, and affects healthy rhythms.

\section{Q. What are the implications of this for whole-body insulin resistance?}

Skeletal muscle plays a major role in glucose absorption following ingestion of food. Development of skeletal muscle insulin resistance and disruption of mitochondrial rhythm may contribute to wholebody insulin resistance and contribute to the progression of T2DM.

\section{Q. How can these findings help improve the management of type 2 diabetes mellitus in people employed in shift work?}

In shift working, people effectively switch their 'day' and 'night' and we have evidence that this affects natural circadian rhythm and mitochondrial function, and may contribute to disturbed insulin 
sensitivity and resistance. ${ }^{1}$ These investigations have shown that switching to a 2-day night shift, people can develop insulin resistance. It is currently not known what the effect of longer-duration night shift work has on insulin resistance and this need to be studied as longer shift periods may be less detrimental; however, it is clear that short shift-work cycles are detrimental to metabolic health. Increased insulin resistance arising from shift-work patterns may lead to increase plasma glucose levels, and consequently increase risk factors for the development of T2DM. Timing of light, food and exercise may be helpful in minimizing the negative consequences of night shift work.

\section{Q. What further studies are planned into the effects of disrupted natural rhythms on insulin resistance?}

Future studies need to examine whether circadian rhythmicity can be restored, post-disruption. The effects of regular exercise, with particular timing in the day, will be important in understanding the capacity to restore natural metabolic rhythmicity. In increasing support for people performing shift work, understanding the roles of exercise and lighting levels will be valuable; increasing bright light during night shifts may help reduce disruption to natural rhythms. $\square$

. Stenvers DJ, Scheer FAJL, Schrauwen P, et al. Circadian clocks and insulin resistance. Nat Rev Endocrinol. 2019;15:75-89.

Tan E, Scott EM. Circadian rhythms, insulin action, and glucose homeostasis. Curr Opin Clin Nutr Metab Care. 2014;17:343-8.

Karthikeyan R, Spence DW, Brown GM, Pandi-Perumal SR. Are type 2 diabetes mellitus and depression part of a common clock genes network? J Circadian Rhythms. 2018;16:4.

Vetter C, Scheer FAJL. A healthy lifestyle - reducing T2DM risk in shift workers? Nat Rev Endocrinol. 2019;15:194-6.

Wefers J, van Moorsel D, Hansen J, et al. Circadian misalignment induces fatty acid metabolism gene profiles and compromises insulin sensitivity in human skeletal muscle. Proc Natl Acad Sci USA 2018:115:7789-94. 